\title{
Structural movies of the gradual spin-crossover in a molecular complex at various physical scales $\dagger$
}

\author{
S. Lakhloufi, ${ }^{a}$ M. H. Lemée Cailleau, ${ }^{b}$ G. Chastanet, ${ }^{a}$ P. Rosa, ${ }^{a}$ N. Daro ${ }^{a}$ and \\ P. Guionneau*a
}

\begin{abstract}
The thermally induced Spin-CrossOver (SCO) undergone by the mononuclear iron(॥) complex [Fe(PM$\left.\mathrm{AzA})_{2}(\mathrm{NCS})_{2}\right]$ (PM = N-2'-pyridylmethylene, AzA = 4-(phenylazo)aniline) is fully pictured by a quasicontinuous structural determination all along the spin-state modification within the sample. This large scale multi-temperature Single-Crystal X-Ray Diffraction (SCXRD) investigation leads to making structural movies. The latter reveal or confirm some features of the SCO that are subsequently validated by the same systematic investigation performed on a zinc isostructural analogue complex. Notably, the continuous views of the temperature dependencies of the unit-cell parameters, the dilatation tensors, the metal coordination sphere geometry and the intermolecular distances confirm a few of the structure property relationships already known for SCO materials. In parallel, the examination of the temperature dependencies of the atomic coordinates and the atomic displacement parameters reveals unexpected behaviours in this gradual SCO material such as antagonistic atomic movements due to the single SCO and the pure thermal effects.
\end{abstract}

\section{Aims and context}

The Spin CrossOver (SCO) phenomenon in iron(II) molecular complexes has been increasingly investigated with both fundamental and application targets. ${ }^{1-4}$ The focus is predominantly on materials that show an abrupt hysteretic SCO for evident reasons due to the property of bi-stability. Clearly, getting iron materials that can be high-spin (HS, $S=2$ ) or low-spin (LS, $S=0$ ) under identical thermodynamic conditions with only a dependence on their history together with a possible switch can appear indeed of large interest in many applicative fields. ${ }^{5-12}$ In contrast, materials that exhibit a gradual SCO in the solid state are generally not considered at all. Notwithstanding, the fundamental investigation of the associated SCO and a re-consideration of their applicative interest should deserve better attention. The purpose of the present work is to give a new insight into the gradual SCO mechanism. To that aim, we propose a quasicontinuous view of the structural property modifications all along the SCO, thanks to a large scale multi-temperature investigation.

\footnotetext{
${ }^{a}$ CNRS, Univ. Bordeaux, ICMCB, UPR9048, 87, Avenue du Docteur Schweitzer, F33600 Pessac, France.E mail: philippe.guionneau@icmcb.cnrs.fr

${ }^{b}$ Institut Laue Langevin, 71 avenue des Martyrs, 33800 Grenoble, France

$\dagger$ Electronic supplementary information (ESI) available: Experimental and crys tallographic data tables, Mossbauer measurements, structural movies at various scales, dilatation tensor matrices, and notes on the positional disorder. CCDC 1492627 1492687. For ESI and crystallographic data in CIF or other electronic format see DOI: $10.1039 / \mathrm{c} 6 \mathrm{cp} 04987 \mathrm{~g}$
}

A multi-temperature investigation is often interesting since it gives information not only on the initial and final phases but also on the mechanism of the transition. This kind of approach is not rare at all in the SCO material field. Numerous examples of structural investigations based on several crystal structures determined in between the temperatures where the sample is purely HS and LS have been reported so far. ${ }^{13-18}$ We propose here to extend this concept to follow almost in a continuous way the SCO by increasing considerably the number of determined crystal structures during the SCO. In the reported studies, this number rarely exceeds ten. In the present work the SCO is described using four times more crystal structures giving a continuous view of the structural modifications associated with the gradual SCO. The superposition of all the crystal structures then allows us to give structural movies of the SCO at different physical scales, from the iron coordination sphere to the crystal packing. In addition to this approach, the zinc analogue of the iron complex studied is investigated by means of the same approach allowing us to very finely separate thermal effects from SCO ones. The overall picture eventually offers a view on the mechanism of the gradual SCO and can certainly be used to probe the structure-property relationships already known for SCO materials. ${ }^{18-21}$

The molecular complex chosen for this investigation has been already reported with iron(II): [M(PM-AzA $\left.)_{2}(\mathrm{NCS})_{2}\right](\mathrm{M}=\mathrm{Fe}$ or $\mathrm{Zn}$, $\mathrm{PM}=N$-2'-pyridylmethylene and AzA $=4$-(phenylazo)aniline $).{ }^{22}$ This iron complex is known to exhibit a gradual SCO and to crystallize in the monoclinic $P 2_{1} / c$ space group in both $\mathrm{HS}$ and LS states. It was previously used to confirm structure-property 
relationships in mononuclear SCO iron(II) materials. ${ }^{23,24}$ The $\mathrm{Zn}$ (II) complex is isostructural to the Fe crystal structure in the HS state and shows no SCO. The use of Zn analogues has already been demonstrated as an optimal choice to witness thermal effects in iron-based materials. ${ }^{25}$

\section{Experimental methodology and details}

Due to the large amount of data obtained from the sixty one investigated crystal structures, usual crystallographic tables are given in the $\mathrm{ESI} \dagger$ and only the experimental protocols are reported below.

\subsection{Synthesis and crystallisation}

The syntheses of both $\left[\mathrm{Fe}(\mathrm{PM}-\mathrm{AzA})_{2}(\mathrm{NCS})_{2}\right]$ and $\left[\mathrm{Zn}(\mathrm{PM}-\mathrm{AzA})_{2}(\mathrm{NCS})_{2}\right]$ follow an already published method using slow diffusion in methanol. ${ }^{22,25}$ The crystallisation process is almost straightforward, and perfectly reproducible and no polymorphism has been observed for these complexes so far. The single-crystal morphology is close to needles for the iron crystals and corresponds to large plates for the zinc ones. The crystal sizes can reach the millimetre scale for both compounds. The crystal colour is dark green for the iron crystals and dark orange for the zinc ones.

\subsection{Magnetism}

The magnetic behaviour of $\left[\mathrm{Fe}(\mathrm{PM}-\mathrm{AzA})_{2}(\mathrm{NCS})_{2}\right]$ and the associated SCO characteristics are already known. ${ }^{22-24}$ They have been however re-determined on MPMS-55 and MPMS-7XL SQUID magnetometers using one unique single-crystal instead of a powder sample or a single-crystal collection as performed previously. The selected crystal has a mass of $0.885 \mathrm{mg}$. The $\chi_{\mathrm{M}} T$ value was determined by cycling temperature between 10 and $310 \mathrm{~K}$ (Fig. 1). No cycle effects are detectable after six successive thermal cycles. The gradual SCO is clearly characterized by a $T_{1 / 2}$ of $189 \mathrm{~K}$. The SCO has already started at about $280 \mathrm{~K}$ and is finished at $105 \mathrm{~K}$. The very large domain of temperature concerned by the SCO makes this compound paradoxically interesting and probably

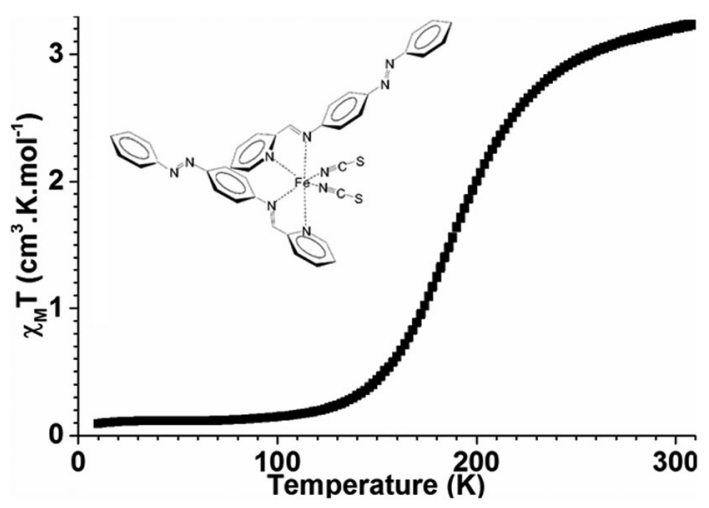

Fig. $1 \chi_{M} T$ as a function of temperature determined on one [Fe(PM $\left.A z A)_{2}(N C S)_{2}\right]$ single crystal. The scheme of the $\left[\mathrm{Fe}(\mathrm{PM} \mathrm{AzA})_{2}(\mathrm{NCS})_{2}\right]$ mole cule is shown in the inset. one of the best candidates to obtain numerous crystal structures in between the pure HS and LS states from a multi-temperature investigation, as targeted in this work. Note that a Mössbauer measurement on a powder sample of $\left[\mathrm{Fe}(\mathrm{PM}-\mathrm{AzA})_{2}(\mathrm{NCS})_{2}\right]$ shows that there is about $5 \%$ of $\mathrm{HS}$ residue at low temperature while the compound is fully HS at room temperature (ESI $\dagger$ ). This HS residue value agrees well with $3.5 \%$ calculated below $50 \mathrm{~K}$ for the single crystal measured by magnetometry, using the room temperature $\chi_{M} T$ value as a reference for the fully HS state. This very low rate of the HS residue has no further incidence on the discussion performed below.

\section{$2.3 \quad \mathrm{X}$-ray diffraction protocol}

As a general matter, keeping in mind that the purpose is to obtain a quasi-continuous structural description all along the thermal SCO, we experienced that some procedures must be absolutely followed to get a final coherent set of data that can lead to appropriate structural movies. Among them is the necessity to manage all the data collections on the same single-crystal, on the same diffractometer and during the same experimental run. The latter condition means no break within the continuity of the temperature cooling (or warming) mode. The experimental strategies must be exactly the same at any temperatures allowing us to extract the same $h k l$ intensity set for each refined crystal structure. Note that this condition facilitates the calculation of the intensities by the collection strategy on the basis of the less symmetric unit-cell in the case of expected symmetry modification in the investigated temperature range. Any deviation from these basic rules leads to discontinuities in the further data analysis and breaks in the film fluidity. Consequences are that the approach described here is time consuming on a laboratory diffractometer and that one has to avoid any interruption during the experiment. As a result, to optimize the experimental time, only datasets with a resolution lower (about $0.9 \AA$ ) than the standard one $(0.8 \AA)$ were recorded here to account for the multi-temperature investigation. This choice is taken into account in the discussion of the results. Note that only routine crystallographic software was used in the present study.

A single crystal of $\left[\mathrm{Fe}(\mathrm{PM}-\mathrm{AzA})_{2}(\mathrm{NCS})_{2}\right]$ was selected and mounted on a glass fibre. The large dimensions of the crystal, $1.2 \times 0.2 \times 0.2 \mathrm{~mm}^{3}$, are not optimal for accurate SCXRD experiments. However, this was not the target here and more importantly this large diffracting volume was necessary to reduce the overall experimental time to a reasonable scale. The sample was mounted on a Kappa Nonius-Brüker diffractometer $(\lambda=$ $0.71071 \AA$ ) equipped with an Oxford Cryosystem nitrogen cryostat and 39 datasets were recorded between 295 and $105 \mathrm{~K}$. The same strategy was used for all the data collections: $136^{\circ} \omega$-scan, 50 s/image, $2^{\circ}$ oscillation per image and a crystal-detector distance of $50 \mathrm{~mm}$. The maximum Bragg angle investigated was about $23^{\circ}$ resulting in a resolution of $0.91 \AA$ with a completeness of $80 \%$. The diffracting quality of the $\left[\mathrm{Zn}(\mathrm{PM}-\mathrm{AzA})_{2}(\mathrm{NCS})_{2}\right]$ crystals is better than for the Fe analogue. A crystal of size $0.60 \times 0.60 \times$ $0.45 \mathrm{~mm}^{3}$ was mounted on the same diffractometer and 21 datasets were recorded: $90^{\circ} \omega$-scan, $40 \mathrm{~s} /$ image, $1^{\circ}$ oscillation per image and a crystal-detector distance of $40 \mathrm{~mm}$. The maximum 
Bragg angle investigated was about $25^{\circ}$ resulting in a resolution of $0.84 \AA$ with a completeness of $73 \%$. Since the crystal structure of this compound was not reported so far, two full datasets leading to high resolution crystal structures were additionally collected at 290 and $80 \mathrm{~K}$. The latter were used to confirm the vicinity of the $\mathrm{Zn}$ and Fe crystal structures. The comparison of the atomic positions of the $\mathrm{Zn}$ and Fe crystal structures gives a Root Mean Square Deviation (RMSD) of 0.057 at $290 \mathrm{~K}$ and 0.189 at $110 \mathrm{~K}$. Consequently, it is clear that the $\mathrm{Zn}$ compound is structurally close to the iron compound in its HS state and quite different from the iron compound in its LS state.

\subsection{Data analysis and structural movies}

The 60 datasets of the multi-temperature Fe and $\mathrm{Zn}$ studies were used to determine and refine the crystal structures using the WINGX suite. ${ }^{26}$ The resulting crystal structures are of good quality with reliability factors of the same order as higher resolution crystal structures, $R_{\mathrm{obs}} \approx 0.04(\mathrm{Fe})$ and $R_{\mathrm{obs}} \approx 0.05$ $(\mathrm{Zn})$ (see all tables in the ESI $\dagger$ ). The atomic position refinement of the isostructural $\left[\mathrm{Fe}(\mathrm{PM}-\mathrm{AzA})_{2}(\mathrm{NCS})_{2}\right]$ and $\left[\mathrm{Zn}(\mathrm{PM}-\mathrm{AzA})_{2}(\mathrm{NCS})_{2}\right]$ compounds is based on 460 parameters. For the former, data collection allows us to extract about 3000 unique observed intensities and thus a good information/parameter ratio higher than 6. For the $\mathrm{Zn}$ compound this ratio is even better, increasing to about 9. Both ratios are far beyond the basic requirement. Consequently, the obtained crystal structures are largely reliable enough to be discussed in detail. In addition, as shown below in the discussion part, the self-consistency of the structural parameters' temperature dependence validates in return the experimental protocol.

In addition, since XRD gives the average atomic positions within the whole sample volume, it is worth noting that the crystal structures determined along the SCO describe an average view of the crystal packing and molecular structures.

Indeed, the investigated compound undergoes a gradual SCO where HS molecules are randomly substituted by LS ones, consequently there are no domains within the crystal where all molecules are in the same spin state, in contrast to previously reported cases. $^{27}$

The structural movies (ESI $\dagger$ ) are obtained by the dynamical superposition of all the crystal structures.

\section{Results and discussion}

\subsection{Unit-cell}

3.1.1 SCO and thermal effects. The unit-cell temperature dependence confirms the significant modifications that the sample undergoes in the range [290-100 K] (Fig. 2). The measurement in a quasi-continuous mode shows no abrupt modification but a strong anisotropy of behaviours especially in the SCO zone. Such a feature is usual in SCO materials. ${ }^{20}$

Interestingly, the thermal contraction of the unit cell is not negligible as clearly seen outside the SCO zone. In contrast, within the latter, i.e. roughly [260-140 K], the unit-cell parameters and volume variations correspond to the sum of the SCO and

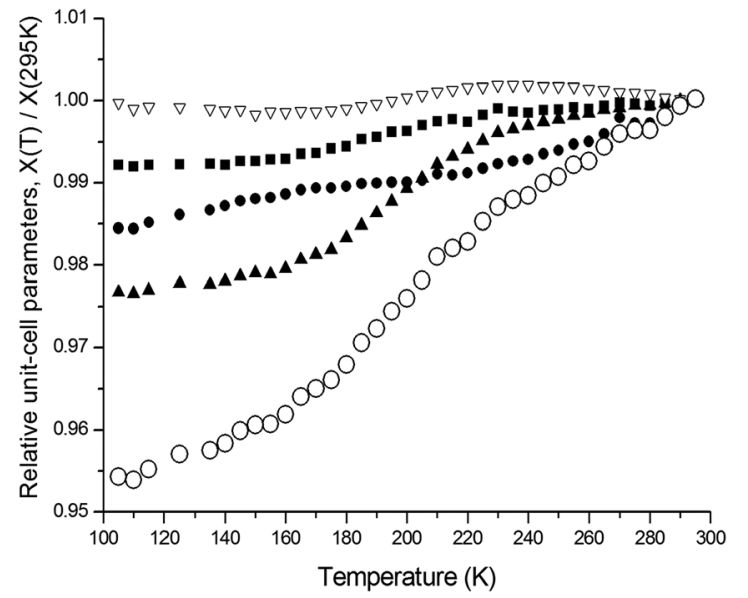

Fig. 2 Normalized unit cell parameters and volume of [Fe(PM AzA $\left.)_{2}(\mathrm{NCS})_{2}\right]$ showing the temperature dependence for a (square), $b$ (small circle), $c$ (full triangle), $\beta$ (empty triangle) and $V$ (empty circle). The reference point is the $295 \mathrm{~K}$ unit cell: $a=15.124(1) \AA, b=14.565(1) \AA, c=17.027(2) \AA, \beta=92.93(2)^{\circ}$ and $V=3745(1) \AA^{3}$.

pure thermal effects. In order to distinguish between them, an elegant solution already used for other compounds ${ }^{27,28}$ is to use the temperature dependence of the isostructural complex where the metal does not undergo any SCO, here the $\mathrm{Zn}$ analogue complex. The comparison between the unit-cell modifications of the iron and zinc complexes clearly evidences the thermal contraction (Table 1). It is then possible, thanks to the vicinity of the unit-cells, to introduce the thermal effects coming from the Zn study in the Fe global modifications to show the modifications of the unit-cell parameters that are due to the SCO only (Fig. 3). In such an approach, the $\mathrm{Zn}$ complex is used as a reference for the Fe compound in its HS state. The comparison of the $\mathrm{Zn}$ and Fe behaviours notably reveals that the large decrease of $c$ is only driven by the SCO (Table 1). It also strongly rescales the unit-cell volume breath, revealing a much smaller SCO effect than suggested without the $\mathrm{Zn}$ comparison. This result puts the studied compound in the SCO material category exhibiting a rather weak volume change (1.6\%) from HS to LS, this volume change having been observed up to $10 \%$ for some materials. ${ }^{20}$

3.1.2 Dilatation tensors in $\mathbf{H S}$ and LS states. If the above strategy reveals the modifications due only to the SCO, the temperature dependence of the unit-cell parameters outside the SCO zone also bears interesting information. The dilatationtensor calculations from these data within the HS domain, [295-260 K], and the LS one, [140-105 K], allow us to compare the

Table 1 Percentage of the unit cell parameters and volume modifications from 295 to $105 \mathrm{~K}$ for $\left[\mathrm{M}(\mathrm{PM} \mathrm{AzA})_{2}(\mathrm{NCS})_{2}\right](\mathrm{M}=$ Fe and $\mathrm{Zn})$. The contribution of the SCO to the structural modifications is calculated considering that the Zn modifications only account for thermal effects while the Fe modifications are the sum of thermal and SCO effects

\begin{tabular}{lrrrrr}
\hline \% of variation & \multicolumn{1}{l}{$b$} & \multicolumn{1}{c}{$b$} & \multicolumn{1}{c}{$\beta$} & \multicolumn{1}{l}{ } \\
\hline M Fe & 0.76 & 1.48 & 2.34 & 0.03 & 4.52 \\
M Zn & 0.25 & 2.57 & 0.02 & +0.79 & 2.93 \\
SCO effects & 0.51 & +1.09 & 2.32 & 0.82 & 1.59
\end{tabular}



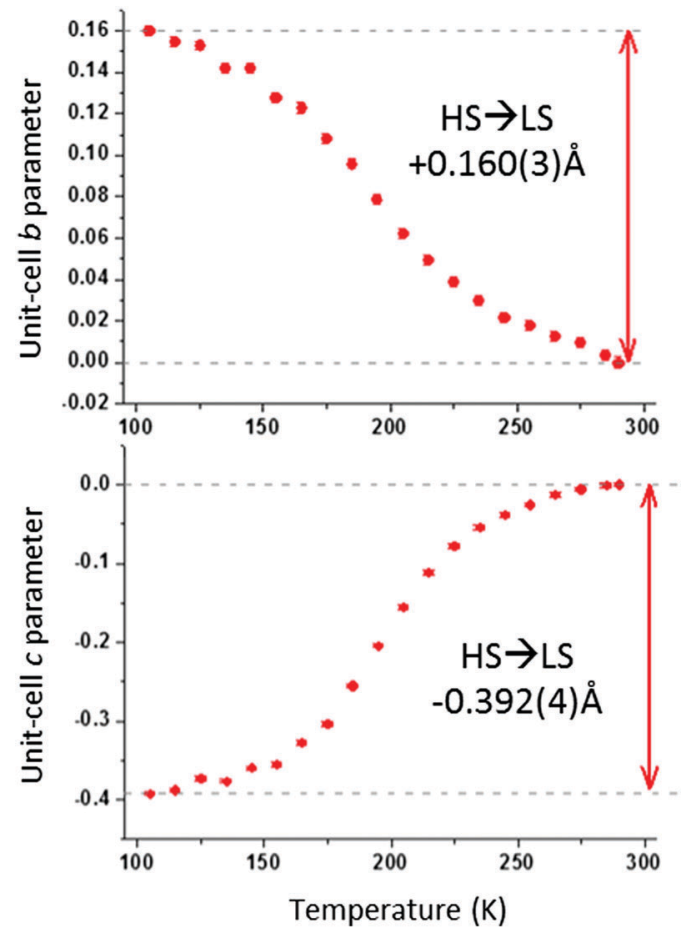

Fig. 3 Modifications ( $\AA$ ) due solely to the SCO for the two selected unit cell parameters $b$ (top) and $c$ (down) in $\left[\mathrm{Fe}(\mathrm{PM} \mathrm{AzA})_{2}(\mathrm{NCS})_{2}\right]$ obtained after the deduction of the pure thermal effects.

rigidity of the HS and LS phases. In such an approach, the $\mathrm{Zn}$ compound is still used as a reference for pure thermal effects. Calculations are performed using the PAScal software. ${ }^{29}$ The three axes of main dilatation are denoted $\mathrm{X} 1, \mathrm{X} 2$ and $\mathrm{X} 3$ and illustrated in Fig. 4. While X1 and X2 correspond to a combination of the parameters $a$ and $c, \mathrm{X} 3$ strictly fits with the $b$ direction, for symmetrical reasons. These directions are very

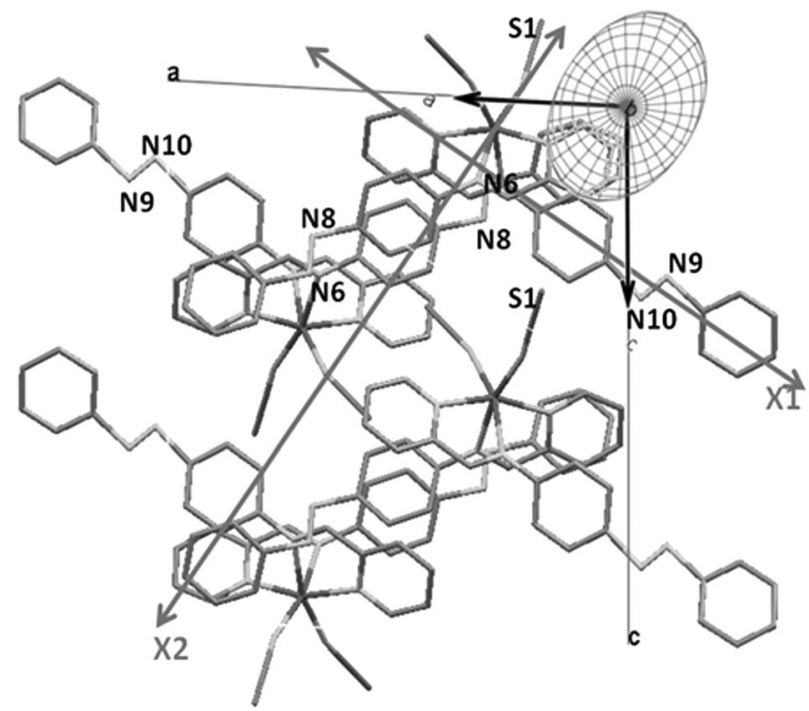

Fig. 4 Representation of the main dilatation directions $X 1$ and $X 2$ within the crystal packing of [Fe(PM AzA $\left.)_{2}(\mathrm{NCS})_{2}\right] . X 3$ corresponds to the unit cell axis $b$.
Table 2 Thermal dilatation coefficients $\alpha_{i}$ for the three main directions of dilatation calculated in the HS and LS temperature domains for [M(PM $\left.A z A)_{2}(N C S)_{2}\right](M=F e$ and $Z n)$. See Fig. 4 for the representation of the directions within the crystal packing

\begin{tabular}{|c|c|c|c|c|}
\hline \multirow{2}{*}{$\begin{array}{l}\text { Dilatation coefficients } \alpha_{i} \\
\left(10^{6} \mathrm{~K}^{1}\right)\end{array}$} & \multicolumn{2}{|c|}{$\underline{\left[\begin{array}{lll}295 & 260 & \mathrm{~K}\end{array}\right]}$} & \multicolumn{2}{|c|}{$\underline{\left[\begin{array}{lll}140 & 105 & \mathrm{~K}\end{array}\right]}$} \\
\hline & $\mathrm{Fe}(\mathrm{HS})$ & $\mathrm{Zn}$ & $\mathrm{Fe}(\mathrm{LS})$ & $\mathrm{Zn}$ \\
\hline$\alpha_{1}$ & $9(8)$ & $28(2)$ & $4(10)$ & 15(1) \\
\hline$\alpha_{2}$ & $56(13)$ & $58(1)$ & $50(7)$ & $31(1)$ \\
\hline$\alpha_{3}$ & $120(14)$ & $164(1)$ & $72(6)$ & $119(2)$ \\
\hline$\alpha_{V}$ & $222(24)$ & $194(2)$ & 129(5) & 135(1) \\
\hline
\end{tabular}

close in the Fe and $\mathrm{Zn}$ compounds and similar in the Fe complex in the HS and LS ranges; the details are given in the ESI. $\dagger$ The values commented here are the coefficients of dilatation, termed $\alpha_{i}$ with $i=1,2,3$ and $V$ corresponding to $\mathrm{X} 1, \mathrm{X} 2, \mathrm{X} 3$ and the whole unit-cell volume, respectively (Table 2). Let us recall that the higher the $\alpha_{i}$ value the easier is the modification of the crystal packing due to a stress along the corresponding direction. Whatever the spin state or the compound ( $\mathrm{Zn}$ or Fe), the direction of main contraction is $\mathrm{X} 3(b)$ while $\mathrm{X} 1$ shows low values and $\mathrm{X} 2$ intermediate ones. The comparison of the $\alpha_{i}$ values shows that the LS crystal structure is more rigid than the HS one since $\alpha_{V}$ is significantly lower in LS than in HS. A network that is more rigid in LS than in HS appears to be a common feature. However, the comparison done here between $\mathrm{Zn}$ and Fe, showing that they are close to each other, reveals that this property is mainly due to pure thermal effects. In contrast, when looking at the details of the dilatation coefficients along the crystallographic directions, some feature of the influence of the SCO on the thermal behaviour can be observed. For example, from HS to LS, the rigidity of the crystal structure significantly increases along the $b$ direction since $\alpha_{3}$ decreases much more for the Fe compound ( $40 \%$ ) than for the $\mathrm{Zn}$ compound ( $28 \%$ ) in the corresponding temperature range. The present approach allows therefore describing the modifications of the crystal-packing rigidity for the investigated sample by introducing a subtle distinction between pure thermal and sole SCO effects. Globally, for this compound, within the investigated temperature range, thermal effects are larger than those due to the SCO.

\subsection{The metal ion and its environment}

3.2.1 Atomic coordinates. The quasi-continuous view of the iron compound behaviour together with the comparison to the zinc one is an approach that can be applied to any structural parameter including the atomic positions. The fractional coordinates of the metal ions (Fig. 5) notably feature the movement of the iron within the investigated temperature range.

Surprisingly, the path from the HS to the LS locations is not necessarily monotonous. When looking at the Fe and $\mathrm{Zn}$ atomic coordinates along the unit-cell parameter $a$, it appears that they strongly decrease with temperature. In the case of Fe however, this decrease is counter-balanced during the SCO that tends to increase this atomic coordinate. As a result, the final lowtemperature coordinates of $\mathrm{Fe}$ and $\mathrm{Zn}$ are rather different along this direction. The Fe path along the SCO within the unit-cell is 

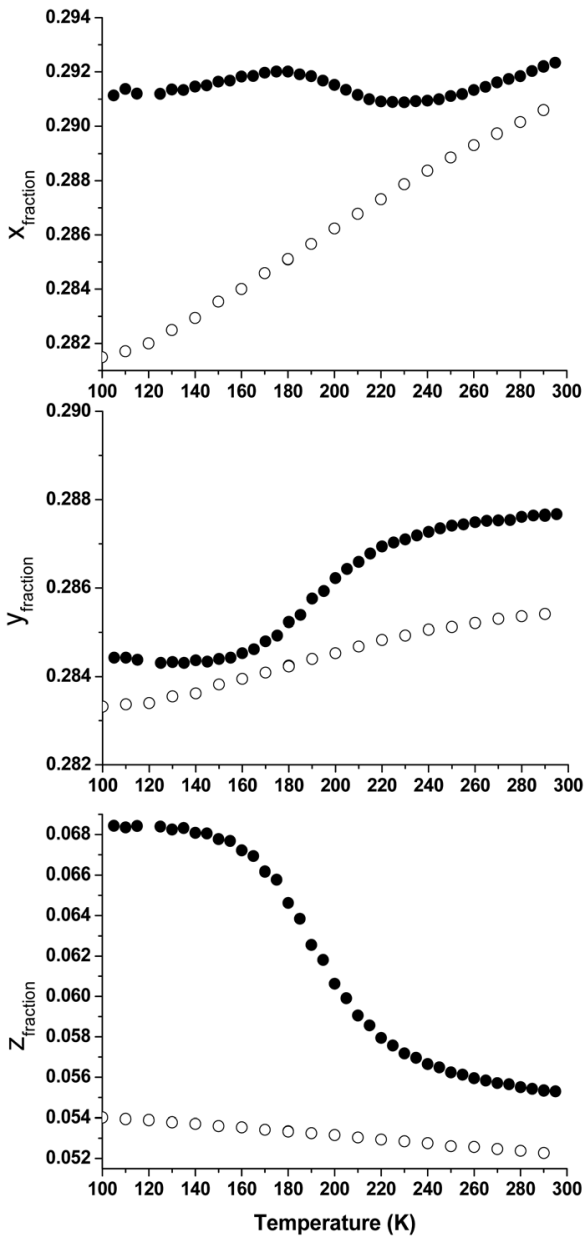

Fig. 5 Fractional coordinates of the metal atom in the unit cell $(x / a, y / b$, $z / c$ ) as a function of temperature for $\left[M(P M A z A)_{2}(N C S)_{2}\right] . M=F e$ (full symbol) and Zn (empty symbol).

therefore not a simple combination of the HS and LS final coordinates.

3.2.2 Metal coordination sphere. It is very well known and documented that the iron coordination sphere geometry is strongly affected by the SCO. ${ }^{19,20,30-32}$ It is not less known that, conversely, the geometry of the HS coordination sphere influences some of the SCO features. ${ }^{18-25,33-37}$ From HS to LS, the Fe-N bond lengths decrease by an average value of $0.2 \AA$, the latter considerably varying depending on the concerned ligands, and the $\mathrm{FeN}_{6}$ octahedron is more regular in the LS state. The metal ion sphere volume strongly decreases at the HS $\rightarrow$ LS SCO and conversely. The present study allows viewing all these modifications (Fig. 6 and 7). Note that the $\mathrm{ZnN}_{6}$ coordination sphere displays no notable modification with temperature showing that the thermal effects are insignificant. For example, the average Fe-N distances for $\left[\mathrm{Fe}(\mathrm{PM}-\mathrm{AzA})_{2}(\mathrm{NCS})_{2}\right]$ goes from 2.154(2) $\AA$ in $\mathrm{HS}$ to $1.960(1) \AA$ in LS. Within the same temperature range, the $\mathrm{Zn}-\mathrm{N}$ distances decrease by less than $0.004 \AA$ settling that thermal effects are negligible. The distortion of the $\mathrm{FeN}_{6}$ coordination sphere measured by the $\Sigma$ parameter ${ }^{20}$ and the corresponding volume $V$ show a continuous decrease from HS to LS that reflects the

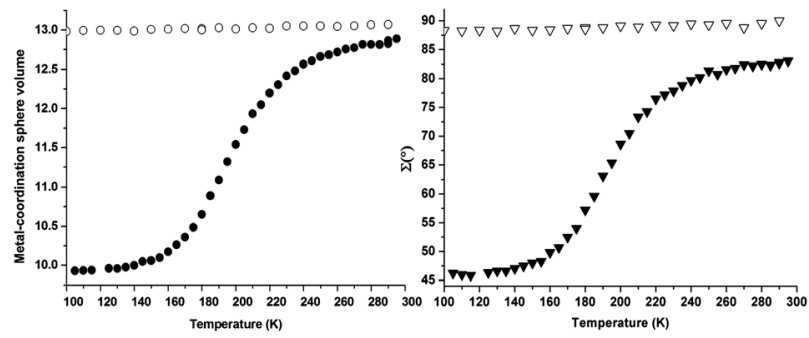

Fig. 6 Modifications of the $M N_{6}$ coordination sphere volume $\left(\AA^{3}\right)$ and distortion $\Sigma\left(^{\circ}\right)$ as a function of temperature for $M=Z n$ (empty symbol) and $M=F e$ (full symbol) in $\left[M(P M A z A)_{2}(N C S)_{2}\right]$.
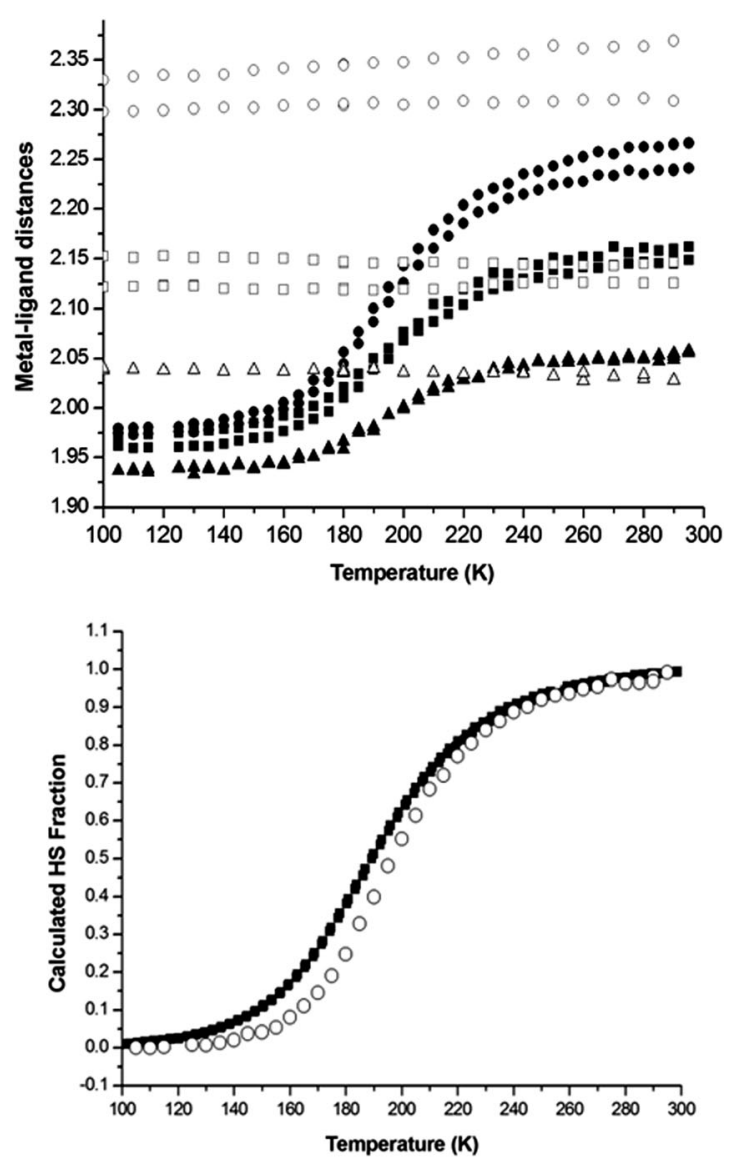

Fig. 7 (Top) Metal ligand $M \quad N$ distances as a function of temperature in $\left[M(P M A z A)_{2}(N C S)_{2}\right]$ with $M=F e$ (full symbols) and $M=Z n$ (empty symbols) referring to thiocyanate (triangles), pyridine (square) and imine (circles) ligands. Comparison (bottom) between the HS fraction calculated from the $\mathrm{Fe} \mathrm{N}$ distances (empty circle) and from the susceptibility curve (full square).

magnetic behaviour. The comparisons of the $\Sigma$ and $V$ values for Fe and Zn clearly confirm the HS $\rightarrow$ LS induced modifications for a $\mathrm{FeN}_{6}$ coordination sphere, respectively, a symmetrisation and a decrease of $3 \AA^{3}(23 \%)$. The superposition of magnetic modifications with structural ones indicates a relatively good agreement between both approaches (Fig. 7). Indeed, the HS fraction has been calculated from the susceptibility curve on one side and from the average of the $\mathrm{Fe}-\mathrm{N}$ distances on the other side, both being compared in Fig. 7. The Fe-N bond lengths cited 
above are used for HS and LS reference values. The temperature of the SCO, $T_{1 / 2}$, is found to be $189 \mathrm{~K}$ using the magnetic measurements and to be $196 \mathrm{~K}$ using the crystallographic data. This gap probably comes from the many differences of the respective experimental protocols. For instance, in a SQUID magnetometer experiment the sample is under vacuum. In an XRD experiment, a shift of a few kelvins for the single-crystal temperature is also not excluded. It appears clearly that differences between SCO temperatures given by structural and magnetic measurements may be a usual feature. The origin of this discrepancy is not fully resolved but is probably connected with single-crystal characteristics such as volume, shape or defects.

The detail of the $\mathrm{Fe}-\mathrm{N}$ distance modification with the temperature and the SCO is given in Fig. 7 confirming that $\mathrm{Fe}-\mathrm{N}$ distances are very close to each other in LS while they strongly differ in HS. In the present case, the $\mathrm{Fe}-\mathrm{N}_{\text {imine }}$ distances decrease by almost $0.3 \AA$ while the $\mathrm{Fe}-\mathrm{N}_{\text {thiocyanate }}$ distances decrease by around $0.1 \AA$, all distances reaching similar values in LS. One of the unexpected features of the $\mathrm{Fe}-\mathrm{N}$ modifications is that the reduction of the $\mathrm{Fe}-\mathrm{N}$ distances is already detectable at $260 \mathrm{~K}$ despite the low conversion rate at this temperature. This confirms the sensitivity of the Fe-N distances to detect the SCO.

\subsection{Molecular and crystal-packing scales}

The superimposition of molecular structures in HS and LS shows some differences beyond the coordination sphere (Fig. 8). The main difference concerns the torsion of the pyridine ligand, the whole contributing to a high average Root-Mean-Square Deviation of 0.202 (max of 0.441) when comparing the HS and LS molecular geometries. The torsion of the pyridine ligand also appears to be significant in the $\mathrm{Zn}$ molecular complex, leading to a RMSD of 0.117 (max of 0.200 ) between the high and low temperature molecular structure geometries. The torsion of the pyridine ligand is certainly a feature that is peculiar to the investigated complex bearing consequently no general information on the SCO phenomenon. It is therefore not further commented here but is certainly related to the positional disorder that affects the AzA ligand (see the ESI $\dagger$ ). In contrast, the thiocyanate ligand geometry appears modified in a mode that may be related to the SCO features. In the $\left[\mathrm{Fe}(\mathrm{PM}-\mathrm{L})_{2}(\mathrm{NCS})_{2}\right]$ compounds, it is well established that the $\mathrm{S}$ atoms are involved in intermolecular contacts that drive

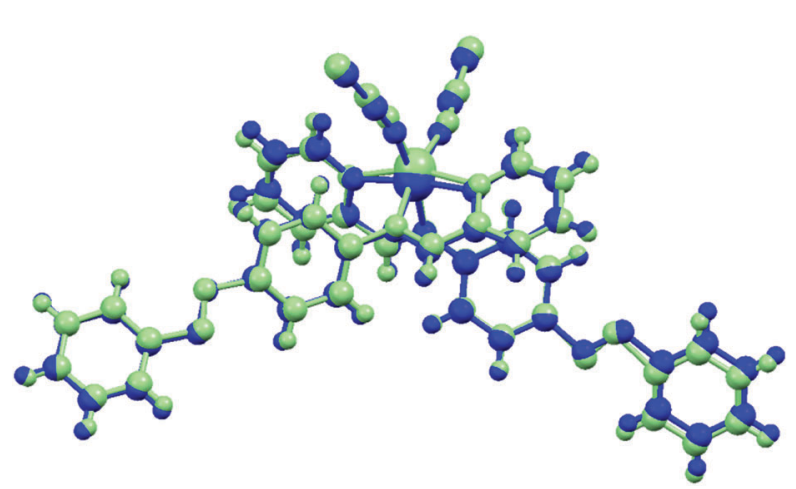

Fig. 8 Superposition of the molecular structures of [Fe(PM AzA $\left.)_{2}(N C S)_{2}\right]$ in $\mathrm{HS}$ at $295 \mathrm{~K}$ (green) and in LS at $105 \mathrm{~K}$ (blue). the abruptness of the SCO; ${ }^{22,23,28}$ the investigated complex being an example of relatively long intermolecular contacts leading to the gradual SCO. This feature is even used in some iron complexes as a basis to control the abruptness of SCO. ${ }^{38}$ Here, looking at the modifications with temperature of the M-NCS angles, the present study reveals that the opening of the Fe-NCS angle from HS to LS contrasts with the closure of the Zn-NCS angle. Between $295 \mathrm{~K}$ and $105 \mathrm{~K}$, the former increases from $166.1(1)^{\circ}$ to $172.5(2)^{\circ}$ when the latter slightly decreases from $166.5(1)^{\circ}$ to $162.5(1)^{\circ}$. The SCO induces effects that are therefore antagonistic to the thermal effects on the MNCS branches. Opposite FeNCS motions induced by SCO and temperature effects have already been observed in the abrupt $\mathrm{SCO}^{39}$ and are therefore evidenced here also for the gradual SCO.

The crystal packing of the title compound has already been described and used to established structure-property relationships in the corresponding $\left[\mathrm{Fe}(\mathrm{PM}-\mathrm{L})_{2}(\mathrm{NCS})_{2}\right]$ family. ${ }^{20}$ Notably, it has been demonstrated that the intermolecular $\mathrm{S} \cdots \mathrm{H}-\mathrm{C}$ interaction plays a paramount role in the SCO features of these complexes. Here, the continuous view of the $\mathrm{S} \cdot \mathrm{H}-\mathrm{C}$ interatomic distances in the $\mathrm{Fe}$ and $\mathrm{Zn}$ compounds shows a regular decrease of these distances from high to low temperature due to both thermal and SCO effects (Fig. 9).

\subsection{Structural movies}

All the above data on the structure-property relationships in $\left[\mathrm{Fe}(\mathrm{PM}-\mathrm{AzA})_{2}(\mathrm{NCS})_{2}\right]$ can be visualized using movies shot thanks to the dynamic superposition of the crystal structures. These movies can be obtained at various physical scales from the coordination sphere to the whole crystal packing (see the ESI $\dagger$ ). Such representation of the temperature dependency is convenient not only to summarize the behaviour of the compound but also to quickly identify modifications. Obviously, it may not be shown within a standard paper article but appears convenient for an oral presentation for example (Fig. 10).

\subsection{Disorder and SCO}

The atomic displacement parameters (ADPs) are among the data determined when solving and refining a crystal structure.

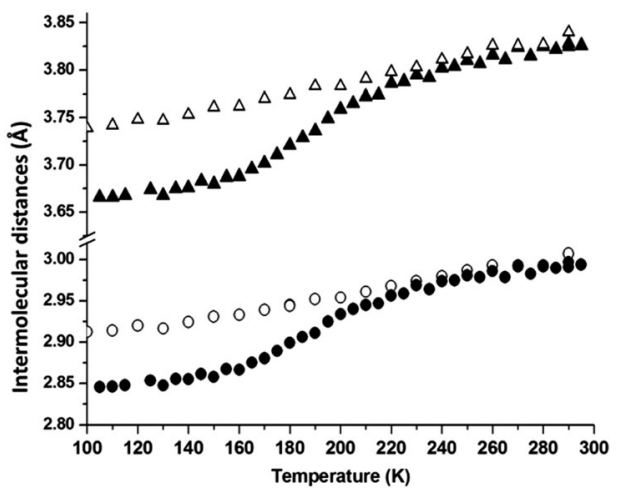

Fig. 9 Temperature dependence of the intermolecular S...H C inter action featured by the $\mathrm{S} \ldots \mathrm{H}(\mathrm{C})$ (circles) and the $\mathrm{S} \ldots \mathrm{C}(\mathrm{H})$ (triangles) short est distances $(\AA \AA)$ within the crystal packing of $\left[M(P M A z A)_{2}(N C S)_{2}\right]$ with $M=$ Fe (full symbols) and $M=Z n$ (empty symbols). 


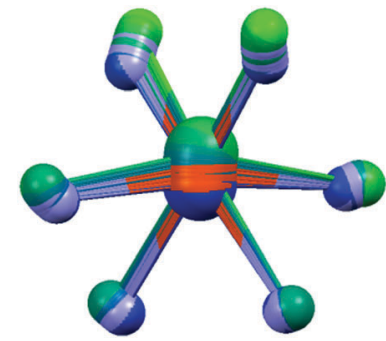

Fig. 10 Superposition of the atomic positions for the $\mathrm{FeN}_{6}$ coordination sphere of the molecule $\left[\mathrm{Fe}(\mathrm{PM} \mathrm{AzA})_{2}(\mathrm{NCS})_{2}\right]$ from the crystal structures determined in the range [105 $295 \mathrm{~K}]$ using green to blue from HS to LS. This static presentation foreshadows the structural movies of the SCO (see the $\mathrm{ESI} \dagger$ ).

It is well known and demonstrated that ADPs contain much more information than the thermal motion of atoms. ${ }^{40}$ In a sense, ADPs encompass every event that moves the atom from its average position within the unit-cell at any physical scales. In other words, ADPs are witnesses of the disorders that affect the single-crystal, from atomic positions to coherent domains. Since the ADPs define an ellipsoid, six values are determined for any atom in a general position within the asymmetric unit. These six parameters can be merged in an isotropic view through the $U_{\text {eq }}$ parameter. The temperature dependence of the latter for three chosen atoms is presented in Fig. 11 for $\left[\mathrm{Fe}(\mathrm{PM}-\mathrm{AzA})_{2}(\mathrm{NCS})_{2}\right]$. The temperature dependence for the $\mathrm{Zn}$ analogue shows a continuous and almost linear decrease of the $U_{\text {eq }}$ parameters with temperature, marking the decrease of the dynamical disorder with temperature. In contrast, for the iron compound, the temperature dependence clearly demonstrates two distinct regimes; one corresponding to the SCO temperature range and the other outside this range. When the sample is purely HS or purely LS, the $U_{\text {eq }}$ parameters decrease with temperature as in the $\mathrm{Zn}$ case. During the SCO however, there is a large plateau that clearly marks an overall increase of the disorder within the sample. The latter gives information on the mechanism of the SCO in the title

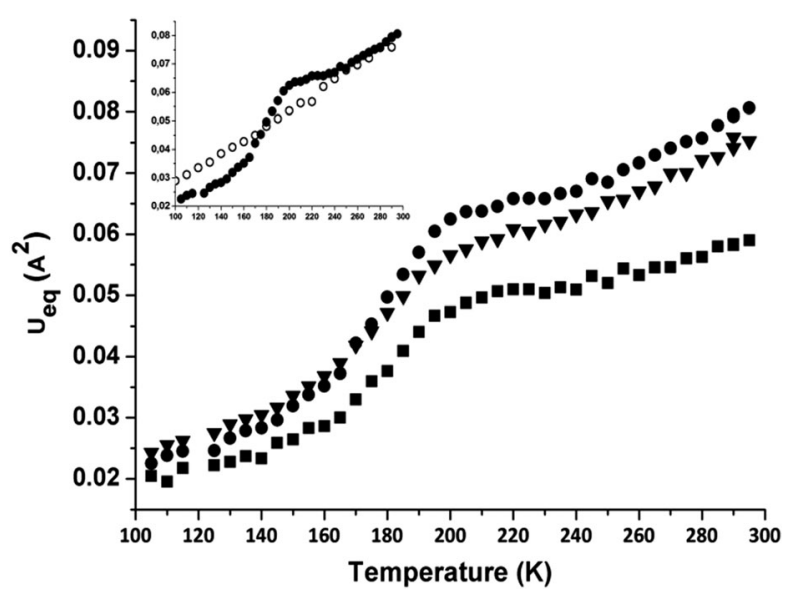

Fig. 11 Temperature dependence of $U_{\text {eq }}\left(\AA^{2}\right)$ in the thiocyanate arms of [Fe(PM AzA) ${ }_{2}(\mathrm{NCS})_{2}$ ] with $\mathrm{N}$ (circle), $\mathrm{C}$ (square) and $\mathrm{S}$ (triangle) atoms. Inset: Comparison of $U_{\text {eq }}\left(\AA^{2}\right)$ of $\mathrm{N}$ for $\left[\mathrm{Fe}(\mathrm{PM} \mathrm{AzA})_{2}(\mathrm{NCS})_{2}\right]$ (full symbol) and $\left[\mathrm{Zn}(\mathrm{PM} \mathrm{AzA})_{2}(\mathrm{NCS})_{2}\right]$ (empty symbol). compound. The high values of $U_{\text {eq }}$ during the SCO characterize the two kinds of molecule geometries within the crystal network, one being HS and the other being LS. The co-existence of the HS and LS molecules does not obey any ordering distribution within the sample. The latter can then be considered as a random substitution of HS molecules by LS molecules (or conversely) and corresponds to an overall increase of the ADP. This behaviour contrasts with the domain mechanism shown by other SCO materials where parts of the sample are made of complexes in the HS state while other parts are made of complexes in the LS state. In the domain mechanism case, the SCO induces a co-existence of the Bragg peaks matching the HS and LS crystal structures but not to an increase of the ADP values. ${ }^{17}$ In the present case, Bragg peaks are single and are positioned in the diffraction images in between their HS and LS positions in parallel with the ADP values that increase during the SCO. Otherwise, note that the global decrease from HS to LS also shows that the pure temperature contribution of the ADP values is largely predominant to any other effects, including the above described disorder.

\section{Summary of movies' lessons on the $\mathrm{SCO}$}

The information given on the structure-property relationships by the combination of the structural movies as defined in this paper appears particularly rich. Of course, at first sight it concerns only the gradual SCO shown by the title compound but some facts could probably be extended to many SCO materials. Above all, the methodology can surely be extended to a wide range of problems.

Therefore, one of the main lessons is the deconvolution of the thermal and SCO effects in the investigated temperature range. For the title compound the volume decreases by $4.5 \%$ from 295 to $100 \mathrm{~K}, 1.6 \%$ being due to the SCO and $2.9 \%$ to thermal effects. SCO and thermal contractions both affect the unit-cell anisotropically but while the former mainly modifies the $c$ parameter, the latter mainly modifies the $b$ parameter. When lowering temperature, the crystal packing is stiffened along $b$ by the SCO while it is stiffened in all directions by thermal effects. Globally, intermolecular contacts are shortened both by SCO and thermal effects resulting in stronger intermolecular interactions between molecular complexes at low temperature in the LS state than at high temperature in the HS state. At the molecular scale, the SCO strongly changes the whole metal coordination sphere geometry while thermal effects have no detectable consequences on it. The significant disorder shown by the molecule is lifted by thermal effects confirming its dynamical origin while the SCO induces a microscopic level disorder coming from the random distribution of HS and LS within the crystal packing.

\section{Conclusions}

The heart of the present results concerns the description of a gradual thermal SCO thanks to an almost continuous structural description all along the temperature domain. This approach brings both pedagogic materials and new scientific knowledge on 
the SCO in a context where this is an increasing requirement. ${ }^{41}$ The former concerns the easy visualisation of the SCO through films, which hardly fits within the form of a written article but which is quite powerful to illustrate conferences or tutorials (see the ESI $\dagger$ ), while the latter corresponds to information on the mechanism of the SCO itself. Notably, it shows that the structural parameters including the atomic positions of the average crystal structure when the sample is part in HS and part in LS cannot be simply deduced by a linear combination of the HS and LS structural parameters. Elsewhere, the fine deconvolution of thermal effects and SCO effects is achieved when the structural movie of an isostructural compound, not showing the SCO, is additionally used. Typically, $\mathrm{Zn}$ analogues can be efficiently used to calibrate the SCO in Fe complexes. Last but not least, the microscopic disorder resulting from the HS to LS modifications is clearly evidenced linking in a whole the different physical scales, from the atomic positions to the coherent domains.

The approach used here is also applicable to any other research field provided that the physical modification investigated is associated with detectable structural changes. In balance, from the experimental time point of view, the sixty crystal structures determined here represent not more than the experimental time necessary a few years ago to collect the data necessary to determine one crystal structure of a similar compound using a point detector. Modern laboratory CCD diffractometers must be used not only to investigate more and more crystals but also to push the frontiers of the structural investigation. Every so often forgotten as a result of the stress on the diffractometer schedules, the present paper proposes a tentative to show that the latter assumption is noteworthy.

\section{Acknowledgements}

We thank Dr Alain Wattiaux (ICMCB) for Mössbauer measurements and Cindy Mauriac (ICMCB) for help on the $\mathrm{Zn}$ analogue crystallisation. S. L. thanks the French government for a $\mathrm{PhD}$ grant. The ANR agency (project ANR femtomat 13-BS04-0002-01) and the Région Aquitaine are acknowledged for funding.

\section{References}

1 Spin-Crossover Materials: Properties and Applications, ed. M. A. Halcrow, John Wiley \& Sons, 2013.

2 S. Hayami, S. M. Holmes and M. A. Halcrow, J. Mater. Chem. $C, 2015,3,7775-7778$ and all included articles.

3 P. Gütlich, A. B. Gaspar and Y. Garcia, Beilstein J. Org. Chem., 2013, 9, 342-391.

4 K. S. Murray, H. Oshio and J. A. Real, Eur. J. Inorg. Chem., 2013, 577-580 and all included articles.

5 O. Kahn and C. Jay Martinez, Science, 1998, 279, 44.

6 P. Gütlich, Y. Garcia and T. Woike, Coord. Chem. Rev., 2001, 219, 839.

7 J. F. Létard, P. Guionneau and L. Goux-Capes, Top. Curr. Chem., 2004, 235, 221.
8 M. D. Manrique-Juárez, S. Rat, L. Salmon, G. Molnár, C. M. Quintero, L. Nicu, H. J. Shepherd and A. Bousseksou, Coord. Chem. Rev., 2016, 308, 395.

9 M. Cavallini and M. Melucci, ACS Appl. Mater. Interfaces, 2015, 7, 16897.

10 O. Roubeau, Chem. - Eur. J., 2012, 18, 15230.

11 G. Aromí, L. A. Barrios, O. Roubeau and P. Gamez, Coord. Chem. Rev., 2011, 255, 485.

12 J. Linares, E. Codjovi and Y. Garcia, Sensors, 2012, 12.

13 D. Chernyshov, M. Hosteller, K. W. Törnroos and H. B. Bürgi, Angew. Chem., Int. Ed. Engl., 2003, 42, 3825.

14 K. W. Törnroos, M. Hosteller, D. Chernyshov, B. Vangdal and H. B. Bürgi, Chem. - Eur. J., 2006, 12, 6207.

15 A. E. Goeta, J. A. K. Howard, A. K. Hughes, D. O'Hare and R. C. B. Copley, J. Mater. Chem., 2007, 17, 485.

16 H. J. Shepherd, P. Rosa, I. A. Fallis, P. Guionneau, J. A. K. Howard and A. E. Goeta, J. Phys. Chem. Solids, 2012, 73, 193.

17 M. Buron-Le-Cointe, J. Hébert, C. Baldé, N. Moisan, L. Toupet, P. Guionneau, J. F. Létard, H. Cailleau and E. Collet, Phys. Rev. B: Condens. Matter Mater. Phys., 2012, 85, 064114.

18 T. A. Pfaffeneder, S. Thallmair, W. Bauer and B. Weber, New J. Chem., 2011, 35, 691.

19 M. A. Halcrow, Chem. Soc. Rev., 2011, 40, 4119.

20 P. Guionneau, Dalton Trans., 2014, 38.

21 M. Shatruk, H. Phan, B. A. Chrisostomo and A. Suleimenova, Coord. Chem. Rev., 2015, 289, 62-73.

22 P. Guionneau, J. F. Létard, D. S. Yuffit, D. Chasseau, J. A. K. Howard, A. E. Goeta and O. Kahn, J. Mater. Chem., 1999, 4, 985.

23 M. Marchivie, P. Guionneau, J. F. Létard and D. Chasseau, Acta Crystallogr., 2005, B61, 25.

24 A. Marino, M. Servol, R. Bertoni, M. Lorenc, C. Mauriac, J. F. Létard and E. Collet, Polyhedron, 2013, 66, 123.

25 F. Le Gac, P. Guionneau, J. F. Létard and P. Rosa, Inorg. Chim. Acta, 2008, 361, 3519.

26 L. Farrugia, J. Appl. Crystallogr., 1999, 32, 837.

27 V. Legrand, S. Pillet, H. P. Weber, M. Souhassou, J. F. Létard, P. Guionneau and C. Lecomte, J. Appl. Crystallogr., 2007, 40, 1076.

28 P. Guionneau, M. Marchivie, G. Bravic, J. F. Létard and D. Chasseau, J. Mater. Chem., 2002, 12, 2546.

29 M. J. Cliffe and A. L. Goodwin, J. Appl. Crystallogr., 2012, 45, 1321.

30 R. T. Acha and M. Pilkington, CrystEngComm, 2015, 17, 8897. 31 C. Boilleau, N. Suaud and N. Guihery, J. Chem. Phys., 2012, 137, 224304.

32 S. Alvarez, J. Am. Chem. Soc., 2003, 125, 6795.

33 C. J. Schneider, J. D. Cashion, N. F. Chilton, C. Etrillard, M. Fuentealba, J. A. K. Howard, J. F. Létard, C. Milsmann, B. Moubaraki, H. A. Sparkes, S. R. Batten and K. S. Murray, Eur. J. Inorg. Chem., 2013, 850.

34 S. O. Schmidt, H. Naggert, A. Buchholz, H. Brandenburg, A. Bannwarth, W. Plass and F. Tuczek, Eur. J. Inorg. Chem., 2016, 2175.

35 Z. Arcis-Castillo, S. Zheng, M. A. Siegler, O. Roubeau, S. Bedoui and S. Bonnet, Chem. - Eur. J., 2011, 17, 14826. 
36 R. J. Wei, B. Li, J. Tao, R. B. Huang, L. S. Zheng and Z. Zheng, Inorg. Chem., 2011, 50, 1170.

37 S. Pillet, E. Bendeif, S. Bonnet, H. J. Shepherd and P. Guionneau, Phys. Rev. B: Condens. Matter Mater. Phys., 2012, 86, 064106.

38 A. Arroyave, A. Lennartson, A. Dragulescu-Andrasi, K. S. Pedersen, S. Piligkos, S. A. Stoian, S. M. Greer, C. Pak,
O. Hietsoi, H. Phan, S. Hill, C. J. McKenzie and M. Shatruk, Inorg. Chem., 2016, 55, 5904.

39 H. J. Shepherd, T. Palamarciuc, P. Rosa, P. Guionneau, G. Molnar, J. F. Létard and A. Bousseksou, Angew. Chem., Int. Ed., 2012, 51, 3910.

40 C. C. Wilson, Crystallogr. Rev., 2009, 15, 3.

41 S. Brooker, Chem. Soc. Rev., 2015, 44, 2880. 